\title{
Characterization of the gut microbiota in hemodialysis patients with sarcopenia
}

\author{
Qifan Zhou ${ }^{1} \cdot$ Hailin Zhang ${ }^{1}$ (C) Lixia Yin ${ }^{1} \cdot$ Guilian $\mathrm{Li}^{2} \cdot$ Wenxue Liang ${ }^{1} \cdot$ Guanjie Chen $^{2}$
}

Received: 27 June 2021 / Accepted: 11 November 2021 / Published online: 29 November 2021

(c) The Author(s) 2021

\begin{abstract}
Purpose Maintenance hemodialysis (MHD) patients are at high risk of sarcopenia. Gut microbiota affects host metabolic and may act in the occurrence of sarcopenia importantly. This study aimed to study the characterization of the gut microbiota in MHD patients with sarcopenia, and to further reveal the complex pathophysiology of sarcopenia in MHD patients. Methods Fecal samples and clinical data were collected from 30 MHD patients with sarcopenia, and 30 age-and-sex-matched MHD patients without sarcopenia in 1 general hospital of Jiangsu Province from December 2020 to March 2021. 16S rRNA sequencing technology was used to analyze the genetic sequence of the gut microbiota for evaluation of the diversity, species composition, and differential microbiota of the two groups.

Results Compared to MHD patients without sarcopenia, the ACE index of patients with sarcopenia was lower $(P=0.014)$, and there was a structural difference in the $\beta$-diversity between the two groups $(P=0.001)$. At the genus level, the relative abundance of Tyzzerella_4 in the sarcopenia group was significantly higher than in the non-sarcopenia group $(P=0.039)$, and the relative abundance of Megamonas $(P=0.004)$, Coprococcus_2 $(P=0.038)$, and uncultured_bacterium_f_Muribaculaceae $(P=0.040)$ decreased significantly.

Conclusion The diversity and structure of the gut microbiota of MHD patients with sarcopenia were altered. The occurrence of sarcopenia in MHD patients may be influenced by gut microbiota.
\end{abstract}

Keywords Maintenance hemodialysis $\cdot$ Sarcopenia $\cdot$ Gut microbiota $\cdot$ Characterization

\section{Introduction}

Sarcopenia is a group of degenerative syndromes involving the accelerated loss of muscle mass and function [1]. Maintenance hemodialysis (MHD) patients are at high risk of sarcopenia due to negative protein balance caused by kidney disease, dialysis treatment, and chronic inflammation combined with dietary restrictions and reduced physical activity $[2,3]$. Sarcopenia reduces the physical function of MHD patients, impairs their ability to conduct daily living activities, and leads to a decline in patients' quality of life. It also increases the risk of hospitalization and hospitalization

Hailin Zhang

342507194@qq.com

1 Lianyungang Clinical College of Nanjing Medical University, The First People's Hospital of Lianyungang, Lianyungang, China

2 The Affiliated Lianyungang Hospital of Xuzhou Medical University, Lianyungang, China costs, and it is an independent predictor of mortality in MHD patients [4-7].

A variety of metabolites produced by the gut microbiota can affect the health of the host $[8,9]$. Previous studies have proposed a potential association between the gut microbiota and sarcopenia, forming the "gut-muscle" axis hypothesis [10-12]. It is believed that the gut microbiota affects the muscle mass and function of the host, by directly affecting the host's inflammatory environment [11], bioavailability of nutrients [13], lipid metabolism [14], and the energy supply [15]. Although these have been predominantly demonstrated in animal models, human studies are limited. No study has directly explored the characteristics of the gut microbiota in MHD patients with sarcopenia.

This study aimed to study the characteristics of the gut microbiota of MHD patients with sarcopenia. The results of this study could potentially shed an additional light on the complex pathophysiology of sarcopenia in MHD patients. 


\section{Materials and methods}

\section{Subjects}

Convenience sampling was used to evaluate whether patients who received MHD treatment at the dialysis units of one general hospitals in Jiangsu Province, China, from December 2020 to March 2021 met the criteria for inclusion and exclusion. This study complied with the Declaration of Helsinki and was approved by the ethics committees of the hospitals where the study was conducted. Written and informed consent was obtained from all participants of the study.

The inclusion criteria were (1) age $\geq 18$ years, (2) ability to communicate normally, (3) hemodialysis for at least 3 months, and (4) dialysis three times a week. Exclusion criteria were (1) taking probiotics, prebiotics antibiotics or laxatives within 3 months before entering the study, (2) inflammatory disease, autoimmune disease, or immunosuppressive treatments, (3) gastrointestinal diseases, diarrhea or constipation, (4) significant changes in diet a week before sampling, (5) serious complications such as liver cirrhosis, hematological diseases, malignant tumor, or serious cardiovascular disease, (6) contraindicated to conduct bioelectric impedance analysis (BIA) test, and (7) history of alcoholism. The control group consisted of MHD patients without sarcopenia, matched for age and sex.

\section{Diagnosis of sarcopenia}

Sarcopenia was diagnosed based on the diagnostic criteria issued by the Asia Working Group for Sarcopenia (AWGS) in 2019 [16]. The criteria included skeletal muscle mass index (SMI), hand grip strength, and $6 \mathrm{~m}$ gait speed. A diagnosis of sarcopenia was made when the patient's SMI decreased along with reduced hand grip strength and/or slow gait speed. The diagnostic threshold of each index was as follows: (1) SMI: male $<7 \mathrm{~kg} / \mathrm{m}^{2}$, female $<5.7 \mathrm{~kg} / \mathrm{m}^{2}$, (2) grip strength: male $<28.0 \mathrm{~kg}$, female $<18.0 \mathrm{~kg}$, and (3) $6 \mathrm{~m}$ gait speed: walking speed $<1 \mathrm{~m} / \mathrm{s}$.

\section{Collection of samples and data}

Fresh feces from fecal centers of all subjects were collected in sterile boxes, and stored at $-80{ }^{\circ} \mathrm{C}$ immediately after sampling, for subsequent analysis. On the day of fecal collection, venous blood was collected before and after dialysis to assay for hemoglobin, serum albumin, total cholesterol, triglyceride, high-density lipoprotein (HDL), low-density lipoprotein (LDL), and CRP levels, as well as KT/V, which is a measure of the adequacy of dialysis. Demographic and clinical data, such as age, gender, comorbidities, dialysis age, BMI, grip strength, and gait speed were collected through a questionnaire. SMI and fat free mass were measured based on BIA technology (InBodyS10, InBody, China) 30-60 min after dialysis.

\section{DNA extraction, PCR amplification and 16S rRNA sequencing}

DNA was extracted according to the instructions of the NucleoSpin Stool kit, (Macherey Nagel, Germany). DNA concentration and purity were detected using a NanoDrop spectrophotometer, and DNA quality was detected by $1.8 \%$ agarose gel electrophoresis. The V3-V4 variable regions of bacterial 16S ribosomal RNA were amplified by PCR with primers $338 \mathrm{~F}$ and $806 \mathrm{R}$; and the PCR products were mixed in equal proportions, and then purified using a DNA purification column (Omega, American). Sequencing was performed using the Illumina MiSeq platform.

\section{Bioinformatics analysis}

The original sequence was assembled and quality-filtered using Flash 1.2.11 and Trimmomatic 0.33, and the chimera was removed using UCHIME 8.1 software to obtain a valid sequence. USearch 10.0 was used to cluster the sequences at the similarity level of $97 \%$ to obtain the operational taxonomic unit (OTU). Diversity was calculated at the OTU level and performed using QIIME2 software. The difference between groups was compared using a $t$ test. The ACE index and Chao 1 index were used to measure the abundance of species. The Simpson index and Shannon index were used to measure the diversity of species, and the coverage index reflected the sequencing depth of the samples. The $\beta$-diversity was calculated according to the Unifrac distance, results of principal coordinate analysis (PCoA), and nonmetric multidimensional scaling (NMDS) with R 3.6.1. The rank sum test was used to analyze the markers of gut microbiota between the two groups.

\section{Statistical analysis}

Epidata 3.1 was used for double-enter of the data, and SPSS 26.0 software was used for statistical analysis. Normal measurement data were expressed as $\bar{x} \pm s$, and two independent samples $t$ test was used for comparison between the groups. Non-normal distribution data were represented by $M\left(P_{25}\right.$, $P_{75}$ ), and comparisons between groups were made using non-parametric test. Countable data were expressed as percentages, and $\chi^{2}$ test was used to compare the two groups. Differences were considered statistically significant when $P<0.05$. 


\section{Result}

\section{General data}

There was no statistically significant differences between the MHD patients with or without sarcopenia in terms of age $(P=0.215)$ and gender $(P=1)$. The LDL $(P=0.005)$, BMI $(P<0.001)$, SMI $(P<0.001)$, fat free mass $(P<0.001)$, grip strength $(P=0.015)$, and gait speed $(P=0.004)$ were significantly lower in MHD patients with sarcopenia. Total cholesterol was significantly higher in MHD patients with sarcopenia $(P=0.016)$ (Table 1$)$.

\section{Analysis of gut microbiota diversity in MHD patients with or without sarcopenia}

$\alpha$-Diversity reflects the abundance and diversity of the microflora in a group. The ACE index in the sarcopenia group decreased significantly $(P=0.014)$ (Fig. 1A). The Chao1 $(P=0.056)$, Simpson $(P=0.815)$ and Shannon index $(P=0.698)$ decreased but not significantly in the sarcopenia group (Fig. 1B, C). The coverage index exceeded 99.9\%, indicating that the number and sequencing depth of the samples could better reflect the community structure of the groups (Fig. 1E).

$\beta$-Diversity reflected the difference in the composition of the gut microbiota between the two groups. The
PCoA diagram showed that PCoA 1 and PCoA2 were significantly separated in the sarcopenia and non-sarcopenia groups, accounting for $20.43 \%$ and $11.1 \%$ of the variation, respectively, along with significant differences in bacterial community compositions $(P=0.001)$ (Fig. 2A). The NMDS graph showed that the difference within the group was small, while the difference between the groups was larger. Stress $=0.1848<0.2$, implying that the analysis was reliable.

\section{Comparison of gut microbiota structure and differential microbiota between MHD patients with and without sarcopenia}

At the genus level, the most dominant bacteria were $\mathrm{Bac}$ teroides (sarcopenia, $15.8 \%$ vs. non-sarcopenia, $17.9 \%$; $P=0.366$ ) and Faecalibacterium (sarcopenia $12.9 \%$ vs. non-sarcopenia $10.7 \% ; P=0.388$ ). The relative abundances of the four genus were significantly different among the groups. The relative abundance of Tyzzerella_4 in the sarcopenia group was significantly higher $(P=0.039)$, and the relative abundance of Megamonas $(P=0.004)$, Coprococcus_2 $(P=0.038)$, and uncultured_bacterium_f_Muribaculaceae $(P=0.040)$, were significantly lower in the sarcopenia group (Fig. 3).
Table 1 Comparison of general data between MHD patients with or without sarcopenia

\begin{tabular}{llll}
\hline Outcomes & Sarcopenia $(n=30)$ & Non-sarcopenia $(n=30)$ & $P$ value \\
\hline Age(year) & $49.9 \pm 12.6$ & $45.87 \pm 12.3$ & 0.215 \\
Gender(man) & $17(56.7 \%)$ & $17(56.7 \%)$ & 1 \\
Complications & & & \\
Diabetes & $2(6.7 \%)$ & $3(10.0 \%)$ & 0.640 \\
Hypertension & $20(66.77 \%)$ & $17(56.7 \%)$ & 0.426 \\
Polycystic kidney & $1(3.3 \%)$ & $0(0 \%)$ & 1 \\
Dialysis age(month) & $36(24.75,76.25)$ & $35.5(16.5,65.25)$ & 0.333 \\
Hemoglobin $(\mathrm{g} / \mathrm{L})$ & $106.23 \pm 13.07$ & $114.23 \pm 17.99$ & 0.054 \\
Serum albumin $(\mathrm{g} / \mathrm{L})$ & $41.03 \pm 2.625$ & $41.66 \pm 3.58$ & 0.329 \\
Total cholesterol $(\mathrm{mmol} / \mathrm{L})$ & $4.47 \pm 0.90$ & $3.90 \pm 0.87$ & 0.016 \\
Triglyceride $(\mathrm{mmol} / \mathrm{L})$ & $1.36(0.82,2.20)$ & $1.31(0.88,1.72)$ & 0.751 \\
HDL $(\mathrm{mmol} / \mathrm{L})$ & $1.09 \pm 0.29$ & $1.23 \pm 0.33$ & 0.093 \\
LDL $(\mathrm{mmol} / \mathrm{L})$ & $1.97 \pm 0.54$ & $2.37 \pm 0.53$ & 0.005 \\
CRP $(\mathrm{mg} / \mathrm{L})$ & $2.61(1.53,4.14)$ & $2.92(1.81,4.99)$ & 0.290 \\
KT/V & $1.53 \pm 0.31$ & $1.43 \pm 0.29$ & 0.309 \\
BMI $\left(\mathrm{kg} / \mathrm{m}^{2}\right)$ & $19.93 \pm 2.70$ & $24.12 \pm 3.94$ & $<0.001$ \\
SMI $(\mathrm{kg} / \mathrm{m} 2)$ & $6.07 \pm 0.86$ & $8.27 \pm 1.60$ & $<0.001$ \\
Fat free mass $(\mathrm{kg})$ & $40.50(31.85,45.28)$ & $54.00(41.33,62.15)$ & $<0.001$ \\
Grip strength $(\mathrm{kg})$ & $27.15(21.25,34.20)$ & $32.25(27.05,44.60)$ & 0.015 \\
Gait speed (m/s) & $0.76 \pm 0.24$ & $0.91 \pm 0.12$ & 0.004 \\
\hline
\end{tabular}

$H D L$ high-density lipoprotein, $L D L$ low-density lipoprotein 
Fig. 1 Analysis of gut microbiota $\alpha$-diversity in MHD patients with or without sarcopenia $* P<0.05$
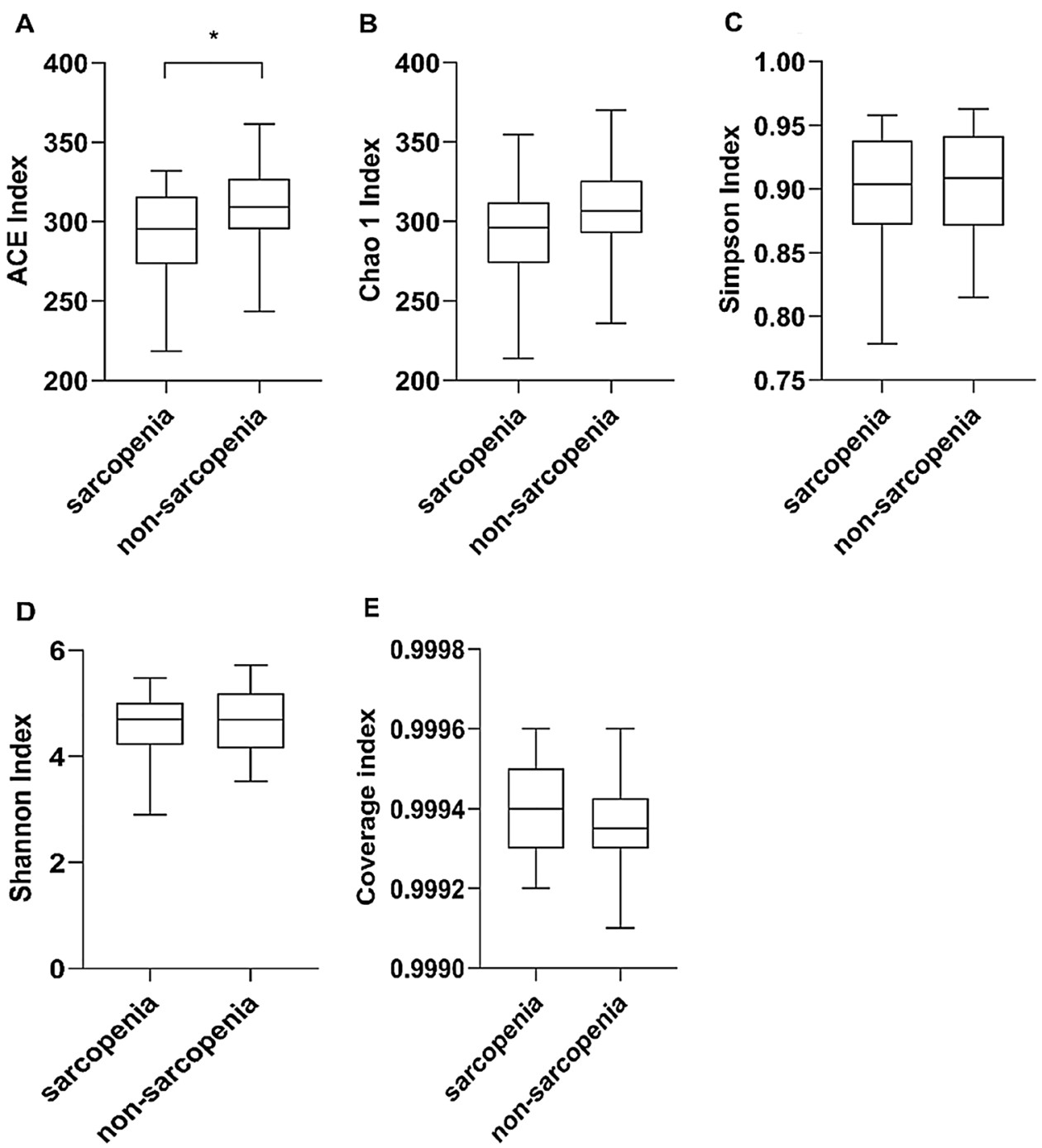
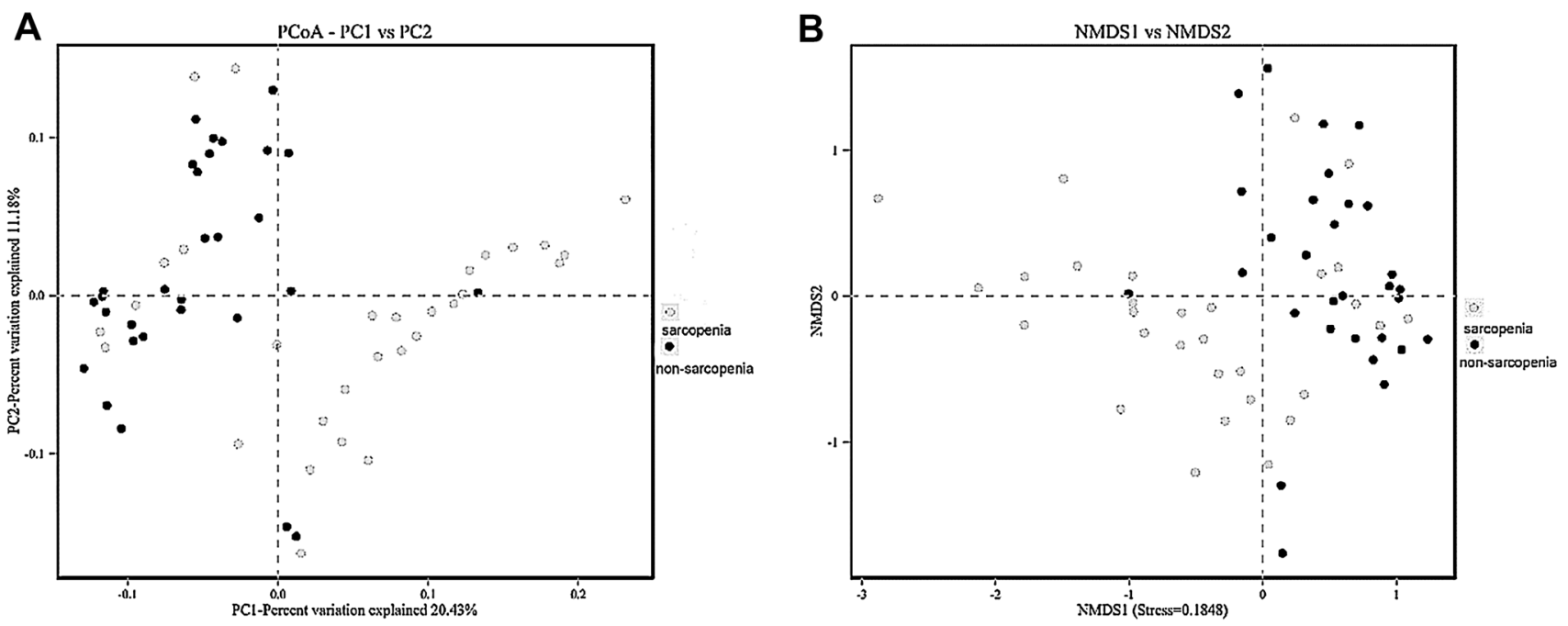

Fig. 2 Analysis of $\beta$-diversity of gut microbiota in MHD patients with or without sarcopenia 
Tyzzerella_4

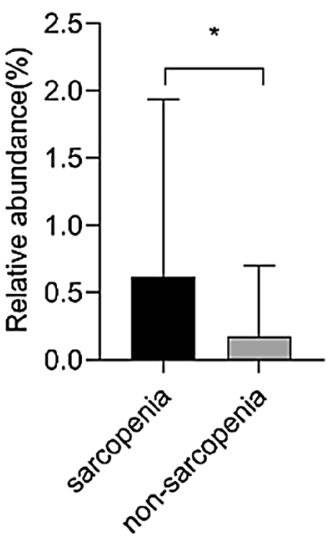

Megamonas

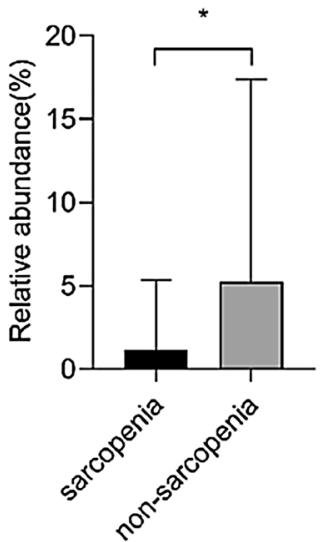

Coprococcus_2

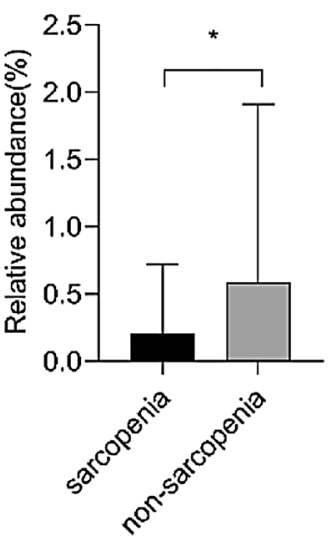

uncultured_bacterium_f_Muribaculaceae

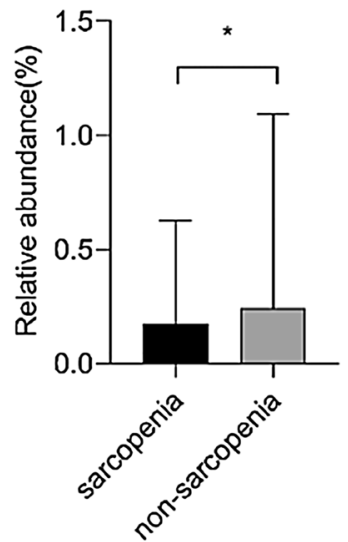

Fig. 3 Analysis of gut microbiota at genus level in MHD patients with or without sarcopenia

\section{Discussion}

The gut microbiota has become the forefront of exploring pathophysiology related to nutritional status and disease states. In this study, 16S rRNA sequencing technology was used to analyze the characteristics of gut microbiota in MHD patients with sarcopenia. The results showed that MHD patients with sarcopenia had lower ACE index compared with MHD patients with non- sarcopenia, and there was a structural difference in the $\beta$-diversity between the two groups. In terms of gut microbiota composition, 4 genera were significantly different between the two groups.

This study found that the diversity of the gut microbiota in the sarcopenia group was significantly lower than that of the non-sarcopenia group, and the difference in the structure of the gut microbiota between the two groups was significant. Previous studies have showed that a stable and diverse gut microbiota is beneficial for the quality and function of skeletal muscle. It was reported that the expression of insulin-like growth factor-1, gene transcription related to skeletal muscle growth, and mitochondrial function significantly decreased leading to skeletal muscle atrophy in germ-free mice, when compared to normal mice colonized by bacteria. After transplanting the gut microbiota of mice with bacteria into germ-free mice, the skeletal muscle mass of the sterile mice increased significantly [17]. In an RCT study, prebiotic supplementation significantly improved the grip strength in frail elderly people [18]. Diversified and stable gut microbiotas dominated by beneficial bacteria can affect energy metabolism through the metabolites produced, thereby improving body composition [19]. Data from this and previous studies, thus, suggest that sarcopenia is closely related to the diversity of the gut microbiota. Decreased diversity and structural instability of the gut microbiota may lead to sarcopenia in MHD patients.
The results of this study showed that compared with MHD patients without sarcopenia, Tyzzerella_4 was significantly higher in MHD patients with sarcopenia while Megamonas, Coprococcus_2, and Uncultured_bacterium_f muribaculaceae were significantly lower.

Tyzzerella_4 is a potential pathogen associated with a poor diet and obesity. In this study, the relative abundance of Tyzzerella_4 in MHD patients with sarcopenia was significantly higher, which may be related to the high-fat diet of MHD patients. Previous studies have found that the relative abundance of Tyzzerella_4 was significantly higher in mice fed a high-fat diet [20]. Similar trends were observed in obese people and people consuming unhealthy diets [21, 22]. A high-fat diet increases body fat tissue, and it is associated with thinner skeletal muscle fibers, increased proteolysis and inflammation in muscle tissue, as well as decreased muscle strength [23, 24]. This may be the influencing factor of Tyzzerella_4 in sarcopenia. Therefore, the gut microbiota may play an important role in the reduction of muscle mass and strength in MHD patients due to diet.

In this study, the abundance of Megamonas and Coprococcus_2 were significantly lower in the sarcopenia group. There are few studies on Megamonas in the sarcopenia population, but many other studies have shown that the relative abundance of Megamonas is significantly reduced in people with low BMI [25-28]. A low BMI indicates malnutrition, and it can easily result in sarcopenia [29]. A previous study showed that Coprococcus_2 was negatively correlated with the frailty index [30], while frailty and sarcopenia were both caused by the decline of multi-system functions such as in aging-related loss of physical functions [31]. This may be because both Megamonas and Coprococcus_2 belong to firmicutes that can produce short chain fatty acids (SCFAs) [32, 33]. SCFAs are products of cellulose, hemicellulose, and other substances that cannot be directly digested by 
gastric digestive enzymes, which are subsequently digested and fermented by the gut microbiota. They have a variety of beneficial effects on muscle quality and function. SCFAs not only improve the permeability of the gut microbiota, but also regulate glucose uptake, reduce insulin resistance and fatty acid oxidation, affect energy supply, plus even regulate immunity and inhibit the secretion of inflammatory response factors [34]. Animal experiments have also directly confirmed the beneficial effects of SCFAs on muscles. Skeletal muscle injury was partially reversed in germ-free mice fed with SCFA [17], and mice with reduced exercise capacity were significantly improved after SCFA supplementation [35]. Therefore, gut microbiota may affect the sarcopenia in MHD patients by manipulating the production of SCFA.

In this study, the abundance of uncultured_bacterium_f $f_{-}$ muribaculaceae in the sarcopenia group was significantly reduced. A previous study found that uncultured_ Bacterium_F_Muribaculaceae was negatively correlated with blood lipid levels [36]. Blood lipids can affect the fatty acid composition of the cell membrane, causing chronic inflammation and insulin resistance. Chronic inflammation promotes protein decomposition, and insulin resistance affects the energy supplies of muscle cells, which eventually leads to a decline in muscle mass and strength [37]. However, there are few studies on uncultured_bacterium_F Muribaculaceae, and further research is needed to understand its role in the pathophysiology of sarcopenia in MHD patients.

This study had several limitations. First, this was a crosssectional study with a small sample size and could not confirm the causal relationship between the gut microbiota and sarcopenia in MHD patients. Secondly, this study only collected sample data from one local region, and the extension of the findings requires further research encompassing a wider region. Finally, this study used $16 \mathrm{~S}$ rRNA amplicon sequencing technology, which limits further research on bacterial colony identification, gene prediction, and functional annotation.

This study showed that the diversity and structure of the gut microbiota in MHD patients with sarcopenia was significantly different from those in MHD patients without sarcopenia, and potentially shed an additional light on the complex pathophysiology of sarcopenia in patients on MHD. A large-sample multicenter interventional study, based on metagenomic sequencing, is needed to establish causal relationships, determine the functional pathways between the gut microbiota and sarcopenia in MHD patients.

Supplementary file2 (JPEG 441 KB)Supplementary Information The online version contains supplementary material available at https:// doi.org/10.1007/s11255-021-03056-6.

Acknowledgements We thank the patients who participated in this study.
Author contribution QZ and HZ designed the study; QZ, LY, GL, and GC performed data collection and analysis; WL did experiments; QZ wrote the manuscript; $\mathrm{HZ}$ edited the manuscript.

Funding This work was supported by the sixth phase of "521 Project" scientific research project in Lianyungang City (LYG06521202123) and Lianyungang City Aging Health Research Project (L202103).

\section{Declarations}

Conflict of interest All authors declare that they have no conflict of interest.

Ethical approval The protocol of this study was approved by the Ethics Committee of the First People's Hospital of Lianyungang, with the approval number KY-20201103001-01.

Informed consent Informed consent was obtained from all individual participants included in the study.

Open Access This article is licensed under a Creative Commons Attribution 4.0 International License, which permits use, sharing, adaptation, distribution and reproduction in any medium or format, as long as you give appropriate credit to the original author(s) and the source, provide a link to the Creative Commons licence, and indicate if changes were made. The images or other third party material in this article are included in the article's Creative Commons licence, unless indicated otherwise in a credit line to the material. If material is not included in the article's Creative Commons licence and your intended use is not permitted by statutory regulation or exceeds the permitted use, you will need to obtain permission directly from the copyright holder. To view a copy of this licence, visit http://creativecommons.org/licenses/by/4.0/.

\section{References}

1. Cruz-Jentoft AJ, Sayer AA (2019) Sarcopenia. Lancet (London, England) 393(10191):2636-2646. https://doi.org/10.1016/S01406736(19)31138-9

2. Sabatino A, Cuppari L, Stenvinkel P, Lindholm B, Avesani CM (2020) Sarcopenia in chronic kidney disease: what have we learned so far? J Nephrol. https://doi.org/10.1007/s40620-020-00840-y

3. Copur S, Sag AA, Afsar B, Rossignol P, Covic A, Kanbay M (2020) Complications of metabolic acidosis and alkalinizing therapy in chronic kidney disease patients: a clinician-directed organspecific primer. Int Urol Nephrol 52(12):2311-2320. https://doi. org/10.1007/s11255-020-02563-2

4. Lin Y-L, Liou H-H, Wang C-H, Lai Y-H, Kuo C-H, Chen S-Y, Hsu B-G (2020) Impact of sarcopenia and its diagnostic criteria on hospitalization and mortality in chronic hemodialysis patients: a 3-year longitudinal study. J Formosan Med Assoc Taiwan Yi Zhi 119(7):1219-1229. https://doi.org/10.1016/j.jfma.2019.10.020

5. Giglio J, Kamimura MA, Lamarca F, Rodrigues J, Santin F, Avesani CM (2018) Association of sarcopenia with nutritional parameters, quality of life, hospitalization, and mortality rates of elderly patients on hemodialysis. J Renal Nutr Official J Council Renal Nutr Nat Kidney Found 28(3):197-207. https://doi.org/10.1053/j. jrn.2017.12.003

6. Bruyère $\mathrm{O}$, Beaudart $\mathrm{C}$, Ethgen $\mathrm{O}$, Reginster $\mathrm{J}-\mathrm{Y}$, Locquet $\mathrm{M}$ (2019) The health economics burden of sarcopenia: a systematic review. Maturitas 119:61-69. https://doi.org/10.1016/j.maturitas. 2018.11.003 
7. Xu W, Chen T, Cai Y, Hu Y, Fan L, Wu C (2020) Sarcopenia in community-dwelling oldest old is associated with disability and poor physical function. J Nutr Health Aging 24(23):339-345. https://doi.org/10.1007/s12603-020-1325-4

8. Lozupone CA, Stombaugh JI, Gordon JI, Jansson JK, Knight R (2012) Diversity, stability and resilience of the human gut microbiota. Nature 489(7415):220-230. https://doi.org/10.1038/natur e11550

9. Gomaa EZ (2020) Human gut microbiota/microbiome in health and diseases: a review. Antonie Van Leeuwenhoek 113(12):2019_ 2040. https://doi.org/10.1007/s10482-020-01474-7

10. Ticinesi A, Nouvenne A, Cerundolo N, Catania P, Prati B, Tana C, Meschi T (2019) Gut microbiota muscle mass and function in aginga focus on physical frailty and sarcopenia. Nutrients. https:// doi.org/10.3390/nu11071633

11. Grosicki GJ, Fielding RA, Lustgarten MS (2018) Gut microbiota contribute to age-related changes in skeletal muscle size, composition, and function: biological basis for a gut-muscle axis. Calcif Tissue Int 102(4):433-442. https://doi.org/10.1007/ s00223-017-0345-5

12. Picca A, Fanelli F, Calvani R, Mulè G, Pesce V, Sisto A, Pantanelli C, Bernabei R, Landi F, Marzetti E (2018) Gut dysbiosis and muscle aging: searching for novel targets against sarcopenia. Mediators Inflamm 2018:7026198. https://doi.org/10.1155/2018/ 7026198

13. Frame LA, Costa E, Jackson SA (2020) Current explorations of nutrition and the gut microbiome: a comprehensive evaluation of the review literature. Nutr Rev 78(10):798-812. https://doi.org/ 10.1093/nutrit/nuz106

14. Du H, Zhao A, Wang Q, Yang X, Ren D (2020) Supplementation of inulin with various degree of polymerization ameliorates liver injury and gut microbiota dysbiosis in high fat-fed obese mice. J Agric Food Chem 68(3):779-787. https://doi.org/10.1021/acs. jafc. 9 b06571

15. Guo N, Wu Q, Shi F, Niu J, Zhang T, Degen AA, Fang Q, Ding L, Shang Z, Zhang Z, Long R (2021) Seasonal dynamics of dietgut microbiota interaction in adaptation of yaks to life at high altitude. NPJ Biofilms Microbiomes 7(1):38. https://doi.org/10. 1038/s41522-021-00207-6

16. Chen L-K, Woo J, Assantachai P, Auyeung T-W, Chou M-Y, Iijima K, Jang HC, Kang L, Kim M, Kim S, Kojima T, Kuzuya M, Lee JSW, Lee SY, Lee W-J, Lee Y, Liang C-K, Lim J-Y, Lim WS, Peng L-N, Sugimoto K, Tanaka T, Won CW, Yamada M, Zhang T, Akishita M, Arai H (2020) Asian working group for sarcopenia: 2019 consensus update on sarcopenia diagnosis and treatment. J Am Med Dir Assoc. https://doi.org/10.1016/j.jamda.2019.12.012

17. Lahiri S, Kim H, Garcia-Perez I, Reza MM, Martin KA, Kundu P, Cox LM, Selkrig J, Posma JM, Zhang H, Padmanabhan P, Moret C, Gulyás B, Blaser MJ, Auwerx J, Holmes E, Nicholson J, Wahli W, Pettersson S (2019) The gut microbiota influences skeletal muscle mass and function in mice. Sci Transl Med. https://doi. org/10.1126/scitranslmed.aan5662

18. Buigues C, Fernández-Garrido J, Pruimboom L, Hoogland AJ, Navarro-Martínez R, Martínez-Martínez M, Verdejo Y, Mascarós MC, Peris C, Cauli O (2016) Effect of a prebiotic formulation on frailty syndrome: a randomized, double-blind clinical trial. Internat J Mole Sci 17(6):5. https://doi.org/10.3390/ijms17060932

19. Chen Y, Zhou J, Wang L (2021) Role and mechanism of gut microbiota in human disease. Front Cell Infect Microbiol 11:625913. https://doi.org/10.3389/fcimb.2021.625913

20. Kang Y, Li Y, Du Y, Guo L, Chen M, Huang X, Yang F, Hong J (2005) Konjaku flour reduces obesity in mice by modulating the composition of the gut microbiota. Int J Obes 43(8):1631-1643. https://doi.org/10.1038/s41366-018-0187-x

21. Liu Y, Ajami NJ, El-Serag HB, Hair C, Graham DY, White DL, Chen L, Wang Z, Plew S, Kramer J, Cole R, Hernaez R, Hou J,
Husain N, Jarbrink-Sehgal ME, Kanwal F, Ketwaroo G, Natarajan Y, Shah R, Velez M, Mallepally N, Petrosino JF, Jiao L (2019) Dietary quality and the colonic mucosa-associated gut microbiome in humans. Am J Clin Nutr 110(3):701-712. https://doi.org/ 10.1093/ajcn/nqz139

22. Jaimes JD, Slavíčková A, Hurych J, Cinek O, Nichols B, Vodolánová L, Černý K, Havlík J (2021) Stool metabolome-microbiota evaluation among children and adolescents with obesity, overweight, and normal-weight using 1H NMR and 16S rRNA gene profiling. PLoS ONE 16(3):e0247378. https://doi.org/10.1371/ journal.pone. 0247378

23. Granic A, Mendonça N, Sayer AA, Hill TR, Davies K, Siervo M, Mathers JC, Jagger C (2020) Effects of dietary patterns and low protein intake on sarcopenia risk in the very old: the newcastle 85+ study. Clinical Nutr (Edinburgh, Scotland) 39(1):166-173. https://doi.org/10.1016/j.clnu.2019.01.009

24. Tallis J, Shelley S, Degens H, Hill C (2021) Age-related skeletal muscle dysfunction is aggravated by obesity: an investigation of contractile function. Implicat Treat Biomole. https://doi.org/10. 3390/biom 11030372

25. Chiu CM, Huang WC, Weng SL, Tseng HC, Liang C, Wang WC, Yang T, Yang TL, Weng CT, Chang TH, Huang HD (2014) Systematic analysis of the association between gut flora and obesity through high-throughput sequencing and bioinformatics approaches. Biomed Res Int 2014:906168. https://doi.org/10. $1155 / 2014 / 906168$

26. Maya-Lucas O, Murugesan S, Nirmalkar K, Alcaraz LD, HoyoVadillo C, Pizano-Zárate ML, García-Mena J (2019) The gut microbiome of Mexican children affected by obesity. Anaerobe 55:11-23. https://doi.org/10.1016/j.anaerobe.2018.10.009

27. Zhang Q, Zou R, Guo M, Duan M, Li Q, Zheng H (2021) Comparison of gut microbiota between adults with autism spectrum disorder and obese adults. PeerJ 9:e10946. https://doi.org/10. 7717/peerj. 10946

28. Palmas V, Pisanu S, Madau V, Casula E, Deledda A, Cusano R, Uva P, Vascellari S, Loviselli A, Manzin A, Velluzzi F (2021) Gut microbiota markers associated with obesity and overweight in Italian adults. Sci Rep 11(1):5532. https://doi.org/10.1038/ s41598-021-84928-w

29. Crossland H, Skirrow S, Puthucheary ZA, Constantin-Teodosiu D, Greenhaff PL (2019) The impact of immobilisation and inflammation on the regulation of muscle mass and insulin resistance: different routes to similar end-points. J Physiol 597(5):1259-1270. https://doi.org/10.1113/JP275444

30. Lim MY, Hong S, Kim JH, Nam YD (2021) Association between Gut Microbiome and Frailty in the Older Adult Population in Korea. J Gerontol A Biol Sci Med Sci. https://doi.org/10.1093/ gerona/glaa319

31. Cesari M, Landi F, Vellas B, Bernabei R, Marzetti E (2014) Sarcopenia and physical frailty: two sides of the same coin. Front Aging Neurosci 6:192. https://doi.org/10.3389/fnagi.2014.00192

32. Sakon H, Nagai F, Morotomi M, Tanaka R (2008) Sutterella parvirubra sp. nov. and Megamonas funiformis sp. nov., isolated from human faeces. Int J Syst Evol Microbiol 58(4):970-975

33. Chevrot R, Carlotti A, Sopena V, Marchand P, Rosenfeld E (2008) Megamonas rupellensis sp. nov, an anaerobe isolated from the caecum of a duck. Int J Syst Evol Microbiol 58(12):2921-2924. https://doi.org/10.1099/ijs.0.2008/001297-0

34. Koh A, De Vadder F, Kovatcheva-Datchary P, Bäckhed F (2016) From dietary fiber to host physiology: short-chain fatty acids as key bacterial metabolites. Cell 165(6):1332-1345. https://doi.org/ 10.1016/j.cell.2016.05.041

35. Okamoto T, Morino K, Ugi S, Nakagawa F, Lemecha M, Ida S, Ohashi N, Sato D, Fujita Y, Maegawa H (2019) Microbiome potentiates endurance exercise through intestinal acetate 
production. Am J Physiol Endocrinol Metab 316(5):E956-E966. https://doi.org/10.1152/ajpendo.00510.2018

36. Mu H, Zhou Q, Yang R, Zeng J, Li X, Zhang R, Tang W, Li H, Wang S, Shen T, Huang X, Dou L, Dong J (2020) Naringin attenuates high fat diet induced non-alcoholic fatty liver disease and gut bacterial dysbiosis in mice. Front Microbiol 11:585066. https:// doi.org/10.3389/fmicb.2020.585066

37. Batsis JA, Mackenzie TA, Jones JD, Lopez-Jimenez F, Bartels SJ (2016) Sarcopenia, sarcopenic obesity and inflammation: Results from the 1999-2004 National Health and Nutrition Examination Survey. Clin Nutr (Edinburgh, Scotland) 35(6):1472-1483. https:// doi.org/10.1016/j.clnu.2016.03.028

Publisher's Note Springer Nature remains neutral with regard to jurisdictional claims in published maps and institutional affiliations. 